\title{
Chronic vulvovaginal candidiasis: characteristics of women with Candida albicans, $C$ glabrata and no candida
}

\author{
A M Geiger, B Foxman, J D Sobel
}

\begin{abstract}
Introduction-Although as many as $5 \%$ of all women complain of chronic vulvovaginitis, little is known about these women. They may often be misdiagnosed and the role of vaginal yeast culture in diagnosing vulvovaginal candidiasis (VVC) among them has not been clearly defined. Methods-To address these deficiencies, we tabulated initial diagnoses among new patients and conducted a medical recordbased, unmatched case-control study among women reporting a history of chronic vulvovaginitis (four or more episodes in the past year) at a vulvovaginitis specialty clinic. Clinical presentation and medical history were compared for women who had a positive vaginal yeast culture for either Candida albicans or $C$ glabrata, or who had a negative culture.
\end{abstract}

Results-One-third of the women had no apparent vulvovaginal disease at their initial visit. All women reported similar symptoms, except for an increased prevalence of painful sexual intercourse in women with $C$ albicans $\left(\chi^{2} \mathbf{p}=0.014\right.$ versus women with $C$ glabrata and $p<$ 0.001 versus women with no candida). Women with $\boldsymbol{C}$ glabrata were more likely to be non-white $\left(\chi^{2} \mathbf{p}=0.071\right.$ compared with women with $C$ albicans) and to report an underlying medical condition $\left(\chi^{2} \mathbf{p} \leqslant 0.001\right.$ versus both women with $C$ albicans and women with no candida). Physical examination was normal only in women with no candida. $C$ albicans cases were more likely to have positive potassium hydroxide microscopy $\left(\chi^{2} \mathbf{p}=\right.$ $0.016)$ and a pH $\leqslant 4.5\left(\chi^{2} \mathrm{p}=0.011\right)$ than were $\boldsymbol{C}$ glabrata cases.

Conclusions-These results suggest that reliance on symptoms and signs alone will result in significant misdiagnosis of chronic vulvovaginitis. Among women with VVC, subtle differences in clinical presentation do not reliably distinguish women with $C$ albicans from those with C glabrata. Our study also indicates that vaginal yeast cultures, while not necessary for every patient, are valuable in confirming negative diagnoses, detecting microscopy false-negatives, and identifying non-C albicans isolates.

(Genitourin Med 1995;71:304-307)
Keywords: candidiasis, vulvovaginal; diagnosis; recurrence

\section{Introduction}

Vulvovaginal candidiasis (VVC) is a syndrome characterised by vulvovaginal inflammation due to vaginal candida infection. Although precise incidence and prevalence estimates are unavailable, there is no question that VVC occurs frequently. In one family practice, $72 \%$ of 204 women aged 18 to 85 years with genitourinary symptoms reported at least one previous episode of VVC. ${ }^{1}$ Among 373 population controls for a cancer study, $44.5 \%$ of women aged 20 to 49 years reported at least one episode of VVC in the past five years; of them, $44.6 \%$ had had five or more episodes. ${ }^{2}$ These studies support the clinical impression that $75 \%$ of women will have at least one episode of VVC during their lives, with $40 \%$ to $50 \%$ experiencing second attacks and a small subset $(\leqslant 5 \%)$ experiencing chronic, recurring episodes. ${ }^{34}$ While women with chronic VVC constitute a small proportion of all women, they may experience symptomatic episodes as often as monthly and may consume disproportionate amounts of clinicians' time. The inability to cure these women quickly and completely is frustrating for both patients and clinicians. ${ }^{45}$

Misdiagnosis of chronic vulvovaginitis due to inadequate patient evaluation may be common, ${ }^{5}$ as signs and symptoms alone are poor diagnostic tools. Diagnostic accuracy is improved with the use of conventional office tests such as saline and potassium hydroxide (KOH) microscopy. ${ }^{16-12}$ However, even if these tests are done properly they may fail to distinguish women with VVC from women with another or no vulvovaginal condition. Vaginal yeast culture may also be useful and is especially indicated in two groups of patients: (1) women with signs and symptoms suggestive of VVC but with negative microscopy and (2) women with positive microscopy who fail to respond to standard therapies who could be infected by non- $C$ albicans species that may require more intensive or alternative therapeutic approaches. ${ }^{4581113-20}$

In this medical record review study of women seen at a vulvovaginitis specialty clinic, we describe the initial diagnoses among women reporting a history of chronic vulvovaginitis and evaluate whether presenting signs, symptoms, office laboratory procedures, and medical history distinguish 
between groups identified by yeast culture. Specifically, we compare women reporting a history of chronic vulvovaginitis (four or more episodes in the past year) who received one of three initial diagnoses at the clinic: (1) acute VVC due to $C$ albicans, (2) acute VVC due to C glabrata (formerly Torulopsis glabrata), and (3) women who appeared normal and had a negative vaginal culture for candida.

\begin{abstract}
Methods
For our unmatched case-control study, we retrospectively reviewed the mediçal records of women who sought care from the head of a clinic specialising in the diagnosis and treatment of chronic vulvovaginal complaints. Women attending this clinic are referred by their clinician or refer themselves after multiple episodes of vulvovaginitis that do not respond to conventional therapies. Prior to their first visit women are asked to cease using all vaginal medications for approximately six weeks, to facilitate diagnosis and minimise any ambiguities in the physical examination due to hypersensitivity reactions to the medications.

Each woman's initial visit to the clinic includes collection of a detailed medical history and a thorough pelvic examination, including bimanual palpation, measurement of $\mathrm{pH}$, amine testing, and microscopic examination with both $10 \% \mathrm{KOH}$ and normal saline. A swab sample from the middle third of the vagina is placed on Sabouraud dextrose agar plates, which are incubated in $\mathrm{CO}_{2}$. Identification of positive yeast cultures is based on germ-tube formation in human serum, chlamydospore production, and the results of carbohydrate assimilation profile tests (API-20C, Sherwood Medical, Plain-
\end{abstract}

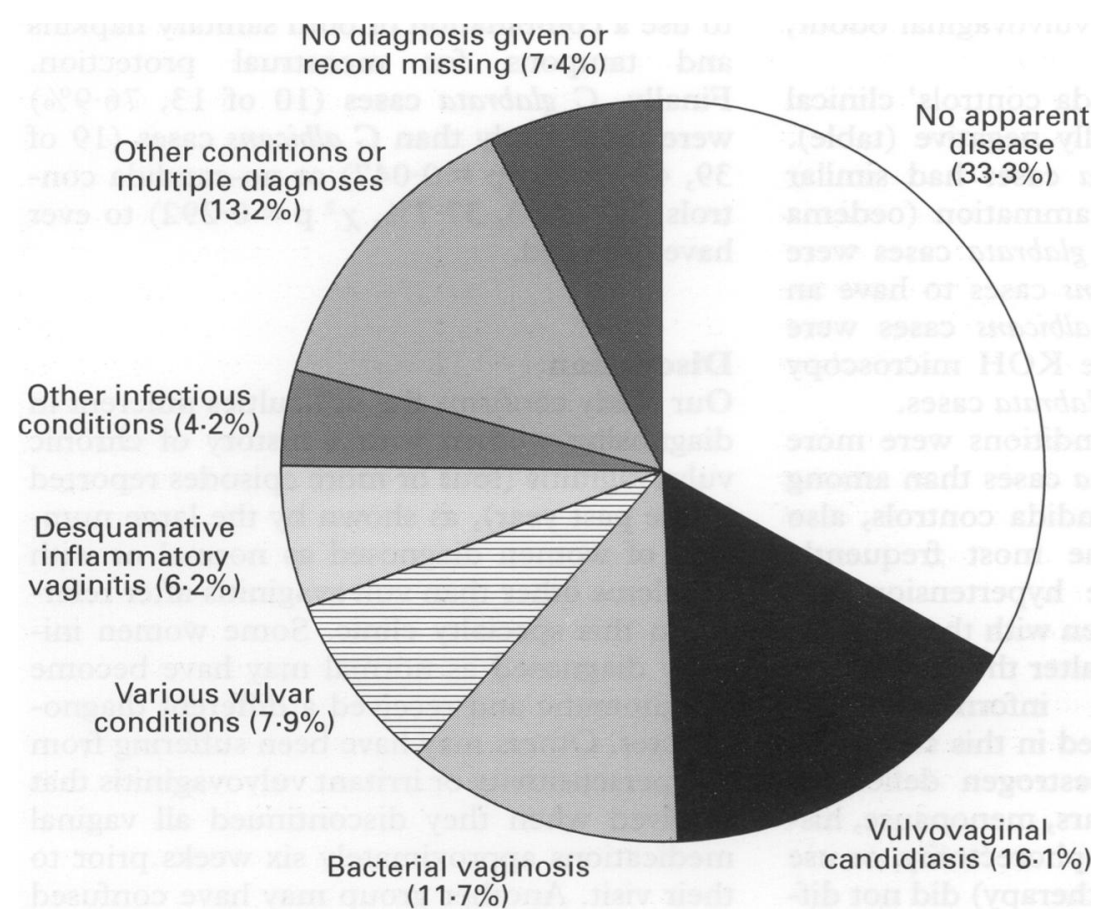

Distribution of Initial Diagnoses in a Vulvovaginitis Specialty Clinic $(n=403)$, fanuary 1991 to March 1992 view, NY). As indicated by examination, additional tests to exclude other vulvovaginal pathogens are conducted.

A case was defined as any symptomatic woman who received a diagnosis of vulvovaginitis on physical examination and had a positive vaginal yeast culture, with other causes of vulvovaginitis excluded. Cases were separated into two groups depending on the organism identified from the cultures, either $C$ albicans or $C$ glabrata. Controls consisted of women who had few or no presenting symptoms and were diagnosed as normal on physical examination after having no findings consistent with VVC or any other cause of vulvovaginitis. Controls also had negative microscopy and vaginal yeast cultures. Cases and controls all reported four or more episodes of vulvovaginitis in the past year. All $48 C$ albicans cases and a random sample of 64 controls who visited the clinic from January 1991 to March 1992 were included, while all 29 Candida glabrata cases identified since the start of the clinic in 1985 were used. No cases or controls were pregnant at the time of their initial visit.

Data were abstracted from a standard clinic intake form completed by the physician at each patient's initial visit. We collected information on demographic characteristics; menstrual, sexual, gynaecological and medical history; current complaints; physical examination results; and the diagnosis and treatment prescribed. Owing to the small sample size, we limited our statistical analysis to two-way $\chi^{2}$ tests and Fisher exact tests when the $\chi^{2}$ expected cell size criteria were violated; both were calculated using SAS. ${ }^{21}$ The study protocol was reviewed and approved by the Human Subjects Review Committee of the University of Michigan School of Public Health.

\section{Results}

INITIAL DIAGNOSES AMONG THE CLINIC POPULATION

The initial diagnoses of 403 new patients with chronic vulvovaginal complaints examined by any provider at the vulvovaginitis specialty clinic from January 1991 to March 1992 are shown in the fig. Although all women reported four or more episodes of vulvovaginitis in the past year, only about $30 \%$ were diagnosed with VVC or bacterial vaginosis, the most common forms of vulvovaginitis. Onethird of women had no apparent vulvovaginal disease at their initial visit and were considered normal. The remaining women received many different diagnoses, including uncommon diseases such as desquamative inflammatory vaginitis and vulvar conditions like vulvar vestibulitis. The remaining results refer only to patients examined by the head of the clinic (JDS), who examines approximately twothirds of all patients at their initial visit.

\section{Case-control comparisons}

$C$ albicans cases $(\mathrm{n}=48), C$ glabrata cases $(n=29)$ and no-candida controls $(n=64)$ were similar with respect to age, marital status 
Characteristics of women reporting a history of chronic vulvovaginitis, by diagnosis at a vulvovaginitis specialty clinic

\begin{tabular}{|c|c|c|c|c|c|c|c|c|c|}
\hline & \multicolumn{2}{|c|}{$\begin{array}{l}\text { Candida albicans } \\
\text { vulvovaginal } \\
\text { candidiasis } \\
(n=48)\end{array}$} & \multicolumn{2}{|c|}{$\begin{array}{l}\text { Candida glabrata } \\
\text { vulvovaginal } \\
\text { candidiasis } \\
(n=29)\end{array}$} & \multicolumn{2}{|c|}{$\begin{array}{l}\text { No apparent } \\
\text { vulvovaginal disease } \\
\text { and no candida } \\
(n=64)\end{array}$} & \multirow{2}{*}{$\begin{array}{l}\text { Candida } \\
\text { albicans } \\
\text { versus } \\
\text { Candida } \\
\text { glabrata } \\
\text { p† }\end{array}$} & \multirow{2}{*}{$\begin{array}{l}\text { Candida } \\
\text { albicans } \\
\text { versus no } \\
\text { candida } \\
\text { p† }\end{array}$} & \multirow{2}{*}{$\begin{array}{l}\text { Candida } \\
\text { glabrata } \\
\text { versus no } \\
\text { candida } \\
\text { p† }\end{array}$} \\
\hline & $N$ & $\%^{*}$ & $N$ & $\% *$ & $N$ & $\%^{*}$ & & & \\
\hline \multicolumn{10}{|l|}{ Demographic Characteristics: } \\
\hline Age $>45$ years & 7 & $14 \cdot 6$ & 5 & $17 \cdot 2$ & 10 & $15 \cdot 6$ & 0.755 & 0.879 & $0 \cdot 844$ \\
\hline White race & 34 & $75 \cdot 6$ & 10 & $52 \cdot 6$ & 43 & $69 \cdot 4$ & 0.071 & 0.481 & $0 \cdot 180$ \\
\hline Currently married & 24 & $51 \cdot 1$ & 12 & $\mathbf{4 4 \cdot 4}$ & 30 & $46 \cdot 9$ & 0.583 & 0.663 & 0.832 \\
\hline Employed & 40 & $83 \cdot 3$ & 20 & $80 \cdot 0$ & 54 & $85 \cdot 7$ & $0 \cdot 724$ & $0 \cdot 730$ & 0.509 \\
\hline \multicolumn{10}{|l|}{ Symptoms: } \\
\hline Duration $\geqslant 1$ year & 41 & $93 \cdot 2$ & 14 & $70 \cdot 0$ & 46 & $79 \cdot 3$ & $0.022 \ddagger$ & 0.050 & $0 \cdot 394$ \\
\hline Discharge & 42 & $93 \cdot \overline{3}$ & 23 & $88 \cdot 5$ & 55 & $96 \cdot 5$ & $0.662 \ddagger$ & $0.652 \ddagger$ & $0 \cdot 175 \ddagger$ \\
\hline Itch & 38 & $88 \cdot 4$ & 23 & $85 \cdot 2$ & 39 & $72 \cdot 2$ & $0 \cdot 726 \ddagger$ & 0.051 & $0 \cdot 194$ \\
\hline Painful sexual intercourse & 27 & $73 \cdot 0$ & 10 & $41 \cdot 7$ & 16 & $32 \cdot 7$ & 0.014 & $<0.001$ & $0 \cdot 450$ \\
\hline \multicolumn{10}{|l|}{ Clinical Presentation: } \\
\hline Vulvar inflammation & 31 & $66 \cdot 0$ & 11 & $57 \cdot 9$ & 50 & $8 \cdot 50$ & 0.538 & $<0.001 \$$ & $<0.001 \mp$ \\
\hline Vaginal inflammation & 29 & $64 \cdot 4$ & 12 & $57 \cdot 1$ & 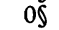 & $0.0 \AA$ & 0.569 & $<0.001 \S$ & $<0.0015$ \\
\hline $\mathrm{pH}>4.5$ & 8 & $17 \cdot 8$ & 10 & $47 \cdot \overline{6}$ & 6 & $9 \cdot 8$ & 0.011 & 0.233 & $<0.001$ \\
\hline $\mathrm{KOH}$ microscopy positive & 34 & $70 \cdot 8$ & 12 & $42 \cdot 9$ & $0 \S$ & 0.08 & 0.016 & $<0.001 \delta$ & $<0.001 \$$ \\
\hline Underlying medical condition & 9 & $19 \cdot 1$ & 15 & $60 \cdot 0$ & 14 & $22 \cdot 2$ & $<0.001$ & 0.695 & $0.001 \ddagger$ \\
\hline
\end{tabular}

*Due to missing data, the denominator may be less than the total $\mathrm{n}$ given

$+\chi^{2}$ test, except as noted.

$\neq \chi^{2}$ test expected cell size requirements violated, so Fisher exact test used.

$\S$ By definition, few or no controls had these characteristics, so these comparisons are somewhat artificial.

and employment (table). C glabrata cases were somewhat less likely to be white than $C$ albicans cases and no-candida controls; the small sample size prevented this difference from achieving statistical significance at the $\mathrm{p}=$ 0.05 level.

Presenting symptoms differed only slightly between case and control groups (table). Painful sexual intercourse was much more common among $C$ albicans cases than among $C$ glabrata cases or no-candida controls. $C$ albicans cases were also more likely to have had recurring vulvovaginal complaints for a year or more. Discharge and itch were common among both case groups and no-candida controls, although $C$ albicans cases were more likely to report itching than controls. Although a high percentage of missing data $(\geqslant 40 \%)$ limited evaluation of other symptoms, both case groups and controls appeared to report similar levels of vulvovaginal odour, dryness, and burning.

By definition, no-candida controls' clinical presentation was essentially negative (table). $C$ albicans and $C$ glabrata cases had similar levels of vulvovaginal inflammation (oedema and/or erythema), but $C$ glabrata cases were more likely than $C$ albicans cases to have an elevated vaginal $\mathrm{pH}$. $C$ albicans cases were much more likely to have $\mathrm{KOH}$ microscopy positive for yeast than $C$ glabrata cases.

Underlying medical conditions were more common among $C$ glabrata cases than among $C$ albicans cases or no-candida controls, also shown in the table. The most frequently reported conditions were hypertension and diabetes; removal of women with these conditions did not appreciably alter the results presented here. Although information on hormonal status was limited in this study, the occurrence of possible oestrogen deficiency (defined as age over 50 years, menopause, history of hysterectomy or oophorectomy, or use of oestrogen replacement therapy) did not differ between the case groups and controls. Both case groups and controls were similar with regard to their history of sexually transmitted diseases, obstetrical-gynaecological procedures (including surgery), number of pregnancies, and types of deliveries. There were also no significant differences in the types of vulvovaginitis treatments tried previously.

Although evaluation of possible predisposing factors for VVC was difficult because of the clinical nature of the data, some differences emerged. $C$ albicans cases (23 of 30, $76.7 \%$ ) were slightly more likely than $C$ glabrata cases (15 of $23,65.2 \%, \chi^{2} \mathrm{p}=$ 0.359 ) or no-candida controls (24 of 43, $\left.55.8 \%, \chi^{2} \mathrm{p}=0.067\right)$ to have been sexually active in the past year; contraceptive practices were similar among both case groups and controls. No-candida controls (21 of 46, $45.7 \%$ ) were more likely than $C$ albicans cases (eight of $34,23.5 \%, \chi^{2} \mathrm{p}=0.042$ ) and $C$ glabrata cases (one of $18,5 \cdot 6 \%, \chi^{2} \mathrm{p}=0.002$ ) to use a combination of both sanitary napkins and tampons for menstrual protection. Finally, $C$ glabrata cases (10 of $13,76.9 \%$ ) were more likely than $C$ albicans cases (19 of $39,48 \cdot 7 \%, \chi^{2} \mathrm{p}=0.047$ ) or no-candida controls (20 of $53,37 \cdot 7 \%, \chi^{2} \mathrm{p}=0.292$ ) to ever have douched.

\section{Discussion}

Our study confirms the difficulties inherent in diagnosing women with a history of chronic vulvovaginitis (four or more episodes reported in the past year), as shown by the large numbers of women diagnosed as normal or with problems other than vulvovaginitis after referral to this specialty clinic. Some women initially diagnosed as normal may have become symptomatic and received a different diagnosis later. Others may have been suffering from a hypersensitivity or irritant vulvovaginitis that resolved when they discontinued all vaginal medications approximately six weeks prior to their visit. Another group may have confused physiologic leukorrhoea with abnormal discharge, a situation clarified at their clinic visit. 
For all women diagnosed as normal or with a condition other than VVC, a negative yeast culture confirmed that VVC was not the appropriate diagnosis.

Women with $C$ albicans VVC, $C$ glabrata VVC and no candida were virtually indistinguishable by demographic characteristics and history. A complaint of painful sexual intercourse may suggest $C$ albicans VVC, whereas women with underlying medical conditions and a shorter history of vulvovaginal complaints may have $C$ glabrata VVC. These characteristics have not been reported by other researchers. We did not confirm previous reports that women with $C$ glabrata VVC tend to be older. ${ }^{22} 23$

We did not find physical examination to be particularly helpful in differentiating VVC due to $C$ albicans from that due to $C$ glabrata; however, other studies have found that $C$ glabrata cases exhibit fewer and less severe symptoms. ${ }^{23}{ }^{24}$ Reliance on KOH microscopy alone would have incorrectly identified about $30 \%$ of $C$ albicans VVC cases and $57 \%$ of $C$ glabrata VVC cases as negative for yeast. Only vaginal yeast cultures identified these false-negative cases, suggesting that cultures are useful in clarifying the diagnosis when the physical examination is consistent with VVC but $\mathrm{KOH}$ microscopy is negative. The higher proportion of false-negative $C$ glabrata cases may be due to the lack of hyphae formation by this organism, making it more difficult to identify microscopically. ${ }^{25}$ Speciation of positive yeast cultures allowed us to differentiate $C$ albicans and $C$ glabrata VVC; speciation also facilitates the tailoring of therapeutics to the organism. ${ }^{13-20}$

Our study was limited in size and has all the problems inherent in medical record review studies. Further, the study population is susceptible to selection bias due to complex, undefined referral patterns in the broader population; this may limit the generalisability of our results to women attending similar clinics and may create false associations in our comparisons. Moreover, the use of a retrospective case-control study design among a population with a long history of vulvovaginal complaints is problematic in that we cannot guarantee the temporal sequence of any associations and do not have any knowledge about the natural history of vaginal infection with various agents. However, this is the only controlled study to specifically address the role of vaginal yeast cultures in the diagnosis of chronic vulvovaginitis and VVC, and one of few to investigate differences in the clinical presentation of $C$ albicans and $C$ glabrata VVC.

In summary, we found that the presenta- tion of women with a history of chronic vulvovaginitis to a vulvovaginitis specialty clinic was similar among groups receiving different diagnoses. As increasing numbers of women avail themselves of over-the-counter antifungals, it is particularly important that women seeking care for recurring VVC be adequately evaluated, with cultures used to verify the presence of yeasts and to exclude the possibility of infection with non- $C$ albicans organisms.

1 Berg AO, Heidrich FE, Fihn SD, et al. Establishing the cause of genitourinary symptoms in women in a family practice. Comparison of clinical examination and compractice. Comparison of clinical examination and

2 Reed BD, Slattery ML, French TK. The association between dietary intake and reported history of candida vulvovaginitis. F Fam Pract 1989;29:509-15.

3 Hurley R. Inveterate vaginal thrush. Practitioner 1975;215: 753-6.

4 Sobel JD. Candidal vulvovaginitis. Clin Obstet Gynecol 1993;36:153-65.

5 Horowitz BJ. Mycotic vulvovaginitis: a broad overview. Am $\mathcal{F}$ Obstet Gynecol 1991;165:1188-92.

6 Bergman J, Berg AO. How useful are symptoms in the diagnosis of candida vaginitis? $\mathcal{F}$ Fam Pract 1983;16: 509-11.

7 Bleker OP, Folkertsma K, Dirks-Go SIS. Diagnostic procedures in vaginitis. Eur $\mathcal{F}$ Obstet Gynecol Reprod Biol 1989;31:179-83. 8 Friedrich EG. Vaginitis. Am f Obstet Gynecol 1985;152:

9 Kaufman RH, Hammill HA. Vaginitis. Prim Care 1990; 17:115-25.

10 McCormack WM, Starko KM, Zinner SH. Symptoms associated with vaginal colonization with yeast. $\mathrm{Am} \mathrm{f}$ Obstet Gynecol 1988;158:31-3.

11 Reed BD, Huck W, Zazove P. Differentiation of Gardnerella vaginalis, Candida albicans, and Trichomonas vaginalis infections of the vagina. F Fam Pract 1989; 28:673-80.

2 Schaaf VM, Perez-Stable EJ, Borchardt K. The limited value of symptoms and signs in the diagnosis of vaginal infections. Arch Intern Med 1990;150:1929-33.

13 Arilla MC, Carbonero JL, Schneider J, et al. Vulvovaginal candidiasis refractory to treatment with fluconazole. Eur $\mathcal{F}$ Obstet Gynecol Reprod Biol 1992;44:77-80.

14 Cauwenbergh G. Vaginal candidiasis: evolving trends in the incidence and treatment of non-Candida albicans the incidence and treatment of non-Candida albicans
infections. Curr Probl Obstet Gynecol Fertil 1990;8:241-5.

15 Horowitz BJ, Giaquinta D, Ito S. Evolving pathogens in vulvovaginal candidiasis: implications for patient care. f Clin Pharmacol 1992;32:248-55.

16 O'Neill S, Howard J. Recurrent vulvovaginal candidiasis. A gynaecological enigma. Aust Fam Physician 1989;18: 99-102.

17 Ott AK, Ashman RB. Modern perspectives on vaginal candidiasis. Aust Fam Physician 1989;18:695-7.

18 Redondo-Lopez V, Lynch M, Schmitt C, Cook R, Sobel JD. Torulopsis glabrata vaginitis: clinical aspects and susceptibility to antifungal agents. Obstet Gynecol 1990;76: 651-5.

19 Summers PR, Sharp HT. The management of obscure or difficult cases of vulvovaginitis. Clin Obstet Gynecol 1993; 36:206-14

20 White DJ, Johnson EM, Warnock DW. Management of persistent vulvovaginal candidosis due to azole-resistant Candida glabrata. Genitourin Med 1993;69:112-4.

21 SAS [computer software]. Personal Computer Version 6.04. Cary, NC, SAS Institute Inc, 1987.

22 Goldacre MJ, Milne LJR, Watt B, Loudon N, Vessey MP. Prevalence of yeast and fungi other than Candida albicans in the vagina of normal young women. Br $\mathcal{F}$ Obstet Gynaecol 1981;88:596-600.

23 Higashide $\mathrm{K}$, Aman R, Yamamuro O. Clinical characteristics correlated with different fungi causing vulvovaginal mycosis. Mycoses 1988;31:213-8, 223-5.

24 Boquet-Jiménez E, Alvarez San Cristóbal A. Cytologic and microbiologic aspects of vaginal Torulopsis. Acta Cytol 1978;22:331-4.

25 Odds FC. Candida and candidosis. London, Baillière Tindall, 1988.

26 Siapco BJ, Kaplan BJ, Bernstein GS, Moyer DL. Cytodiagnosis of candida organisms in cervical smears. Acta Cytol 1986;30:477-80. 